\title{
The Combination of Pulmonary Vein Electrophysiology and Atrial Fibrosis Determines Driver Location
}

\author{
Caroline H. Roney, Jason D. Bayer, Rémi Dubois, Marianna Meo, \\ Hubert Cochet, Pierre Jaïs, Edward J. Vigmond \\ IHU Liryc, University of Bordeaux, Bordeaux, France
}

\begin{abstract}
Patients exhibit a range of pulmonary vein (PV) and fibrosis properties, but their individual and combined effects on arrhythmia inducibility and maintenance mechanisms is unknown. We aim to predict locations of high phase singularity (PS) density using a combination of PV electrophysiology and fibrosis measurements. We used a bilayer computational human atrial model with interstitial fibrosis based on delayed-enhancement MRI (DE-MRI), including high or low levels of fibrosis. PV action potential duration and conduction velocity were varied. Reentry was observed around the left atrial (LA)-PV junction in a subset of patients and reproduced in the model for PV properties with baseline APD, but not for short. For patients for whom rotors were located away from the PVs, increasing model fibrosis from low to high increased the model agreement with clinical PS density $2.3 \pm 1.0$ fold. For other patients, lower levels of fibrosis with baseline PV properties were more appropriate. These results suggest that modelling both PV electrophysiology and atrial fibrosis is important for determining arrhythmia mechanisms. The combined use of PV and DE-MRI measurements may improve ablation therapy success.
\end{abstract}

\section{Introduction}

Despite low overall success rates for catheter ablation of persistent atrial fibrillation (AF) patients, there is a subset of patients for whom pulmonary vein isolation (PVI) alone represents a successful treatment strategy. However, how to predict whether PVI is sufficient for a given patient with persistent AF [1] and what to do for the majority of patients in whom it is not effective is unknown.

Effective refractory period (ERP) and conduction velocity (CV) in the pulmonary veins (PVs), and their gradients with the left atrial (LA) body, exhibit distinct properties in patients with AF. Paroxysmal AF patients have shorter ERP and longer conduction delays compared to control patients. Patients for whom persistent AF is likely to ter- minate after PVI have a larger ERP gradient compared to those who require further ablation $[1,2]$. In addition, $\mathrm{AF}$ patients show a number of other differences to control patients: conduction delay is increased at the LA/PV junction [2]; and fiber direction may be more disorganised, particularly at the PV ostium [3].

Clinically, electrical driver (rotors or focal sources) location changes with AF duration; drivers are typically located close to the PVs in early AF, but are also located elsewhere in the atria as AF progresses [4]. Atrial fibrosis modifies conduction and is a major factor associated with AF. However, there is conflicting evidence regarding the relationship between driver location and fibrosis distribution $[5,6]$.

The individual contributions of these factors to arrhythmia susceptibility and maintenance are unknown, and difficult to determine clinically. We hypothesise that the combination of PV electrophysiology and atrial body fibrosis determines driver location and likely effectiveness of PVI. We will use computational modelling to gain mechanistic insight into the individual contribution of PV ERP (assessed by changing action potential duration, APD), CV, fiber direction, and fibrosis on arrhythmia susceptibility and dynamics. We aim to incorporate APD and conduction slowing data for the PVs and LA to determine mechanisms underlying arrhythmia susceptibility, by testing inducibility from PV ectopic beats. We also aim to predict driver location, and whether PVI is effective.

\section{Methods}

A previously published bi-atrial bilayer model [7] that incorporates regional heterogeneity in repolarisation and conduction properties was used for these studies. The Courtemanche-Ramirez-Nattel human atrial ionic model was used with changes representing electrical remodelling during $\mathrm{AF}$, with modifications of the conductances to incorporate regional heterogeneity in repolarisation. Parameters for the baseline PV model were taken from Krueger et al. [8].

Arrhythmia inducibility was tested by extrastimulus 
pacing from each of the four PVs individually using a clinically motivated protocol [9], to simulate the occurrence of PV ectopics. Each PV was paced with five beats at a cycle length of $160 \mathrm{~ms}$, and a coupling interval following sinus rhythm in the range $200-500 \mathrm{~ms}$. Thirty-two combinations were tested for each model set up, and the proportion of inducible cases was calculated.

\subsection{PV electrophysiology and fibers}

To investigate the effects of PV electrophysiology on arrhythmia inducibility, the value of the inward rectifier current $\left(\mathrm{I}_{\mathrm{K} 1}\right)$ conductance and tissue level conductivity were varied in the PVs to modify APD and CV respectively, with changes applied either homogeneously or following a ostial-distal gradient. This gradient was implemented by calculating geodesic distances from the rim of nodes at the distal PV boundary to all nodes in the PV and from the rim of nodes at the LA/PV junction to all nodes in the PV. The ratio of these two distances was then used as a distance parameter from the LA/PV junction to the distal end of the PV.

$\mathrm{I}_{\mathrm{K} 1}$ conductance was rescaled by a scaling factor in the range 0.5-2.5, resulting in PV APDs in the range: $100-190 \mathrm{~ms}$. Gradients of $\mathrm{I}_{\mathrm{K} 1}$ conductance ranged from the baseline value at the LA/PV junction, to a maximum scaling factor in the range $1-2.5$ at the distal boundary.

Longitudinal and transverse tissue conductances were divided by $1,2,3$ or 5 , resulting in $\mathrm{CVs}$, measured along the PV axis, in the range: $0.28-0.67 \mathrm{~m} / \mathrm{s}$.

PV fiber direction shows great inter-patient variability. Fiber direction in the four PVs was modified on the endocardium and epicardium according to different reported fiber arrangements $[3,10,11]$ : 1 . circular arrangement on both the endocardium and epicardium; 2 . spiralling arrangement (45 degrees) on both the endocardium and epicardium; 3. circular arrangement on the endocardium, with longitudinal epicardial fibers; 4. fibers progress from longitudinal at the distal vein to circular at the ostium, with identical endocardial and epicardial fibers; 5. epicardial layer fibers as per case 4, with circular endocardial fibers; 6 . as per case 4 , but with a chaotic fiber arrangement at the LA/PV junction. These arrangements are shown in Fig 1.

\subsection{Model phase singularity analysis}

Ten second arrhythmia data were analysed to determine locations of electrical drivers and wavefront breakup. Phase singularities (PSs) for the transmembrane potential data were calculated, and PS spatial density maps were determined using previously published methods [12]. PS density maps were then partitioned into the LA body, PV regions, and the RA to assess whether drivers were located in the vicinity of the PVs.

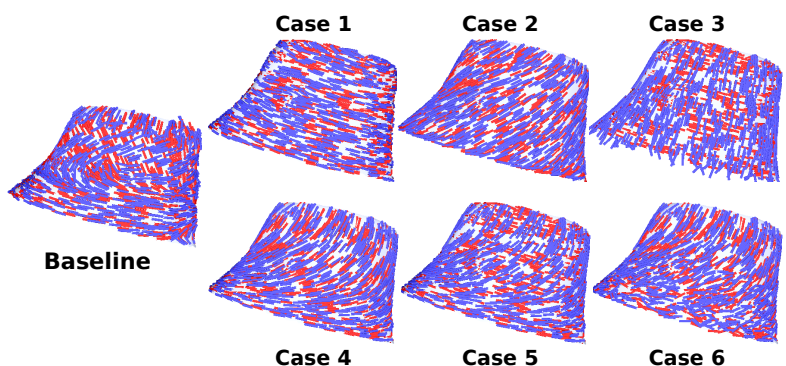

Figure 1. Pulmonary vein fiber directions for the endocardial (red) and epicardial (blue) surfaces.

\subsection{Inclusion of patient fibrosis data and comparison to clinical PS densities}

Interstitial fibrosis was incorporated in the model based on delayed-enhancement MRI, using our previously published methodology [13]. The model arrhythmia dynamics were compared to AF recordings from a commercially available non-invasive ECGi mapping technology (CardioInsight Inc.) for which phase mapping analysis was performed as previously described [14].

\section{Results}

\subsection{PV electrophysiology and fibers}

A difference in APD between the model LA and PVs was required for AF induction. Removing this APD difference by setting the PV components of the model to LA cellular properties resulted in non-inducibility, whereas setting the LA to PV cellular properties resulted in either noninducibility or macrorentry.

Table 1 shows arrhythmia inducibility for PV APD changes incorporated as either a homogeneous change, or following a gradient. Cases for which PV APD was longer than LA APD were non-inducible. Inducibility initially increased as APD decreased below the baseline value (181ms) and then fluctuated. Arrhythmia inducibility was higher for APD varied following a ostial-distal gradient than for homogenous APD, comparing cases with equal distal APD.

PS location varied with PV APD; PV PS density was low in cases of short APD. This change was more noticeable for cases with homogeneous PV APD than for a gradient in APD, as shown in Fig 2, in which PV reentry is observed for the baseline case and heterogeneous APD case, but not for a homogeneous decrease in APD.

Homogeneous CV slowing decreased arrhythmia inducibility (from 0.38 ( 12 out of 32 cases were inducible) at $0.67 \mathrm{~m} / \mathrm{s}$ to 0.03 at $0.28 \mathrm{~m} / \mathrm{s}$ ) since conduction slowing led to an increased interval between extrastimulus beats at the $\mathrm{LA} / \mathrm{PV}$ junction. The effects of $\mathrm{CV}$ on inducibility were 
Table 1. Inducibility ratios for different PV APDs, varied homogeneously $(\mathrm{H})$ or following a gradient $(\mathrm{G})$

\begin{tabular}{ccc}
\hline \hline Distal APD (ms) & Inducibility (H) & Inducibility (G) \\
\hline 190 & 0 & \\
181 & 0.38 & \\
170 & 0.53 & \\
163 & 0.38 & 0.53 \\
143 & 0.31 & 0.41 \\
120 & 0.53 & 0.56 \\
109 & 0.41 & 0.53 \\
100 & 0.41 & \\
\hline \hline
\end{tabular}

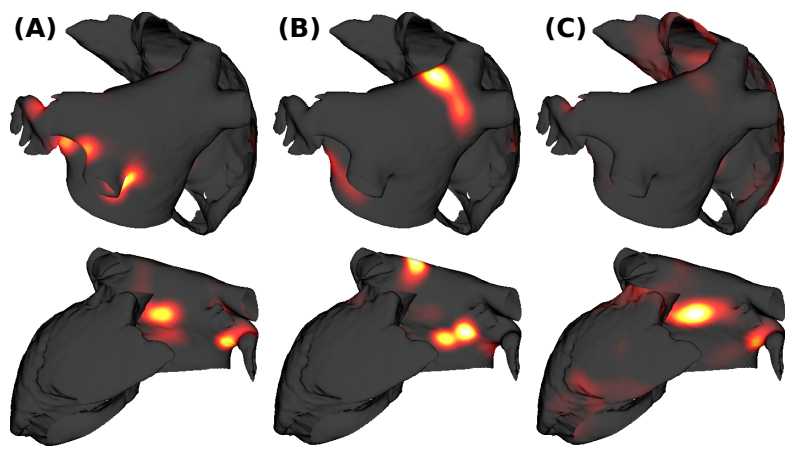

Figure 2. Normalised PS density maps for (A) baseline PV values; (B) $I_{K 1}$ varied following a gradient, resulting in APDs ranging from 163 to $181 \mathrm{~ms}$; (C) $\mathrm{I}_{\mathrm{K} 1}$ varied homogeneously to give a PV APD of $163 \mathrm{~ms}$.

larger than those of APD.

All changes in fiber direction increased inducibility compared to the baseline fiber direction (baseline case: 0.38 ; modified fiber direction cases 1-6: inducibility range $0.53-0.63)$. The greatest inducibility occured for cases with circular fibers at the ostium on both endocardial and epicardial surfaces (cases 1 and 4, 0.63), independent of fiber direction at the distal PV end. This inducibility was reduced if the epicardial fibers were not circular at the ostium (case 3, 0.56), or if fibers were spiralling (case 2, 0.56 ) instead of circular.

\subsection{Fibrosis plus PV properties}

LA fibrosis properties were varied to represent interstitial fibrosis in paroxysmal and persistent AF patients, incorporating average DE-MRI distributions [15] into the model. These control, paroxysmal and persistent AF levels of fibrosis were then combined with PV properties varied as follows: baseline CV and APD $(0.67 \mathrm{~m} / \mathrm{s}, 181 \mathrm{~ms})$, slow CV $(0.51 \mathrm{~m} / \mathrm{s})$, short APD $(120 \mathrm{~ms})$, slow CV and short APD. PS distributions in Fig 3 show that reentry occurred around the LA/PV junction in the case of baseline

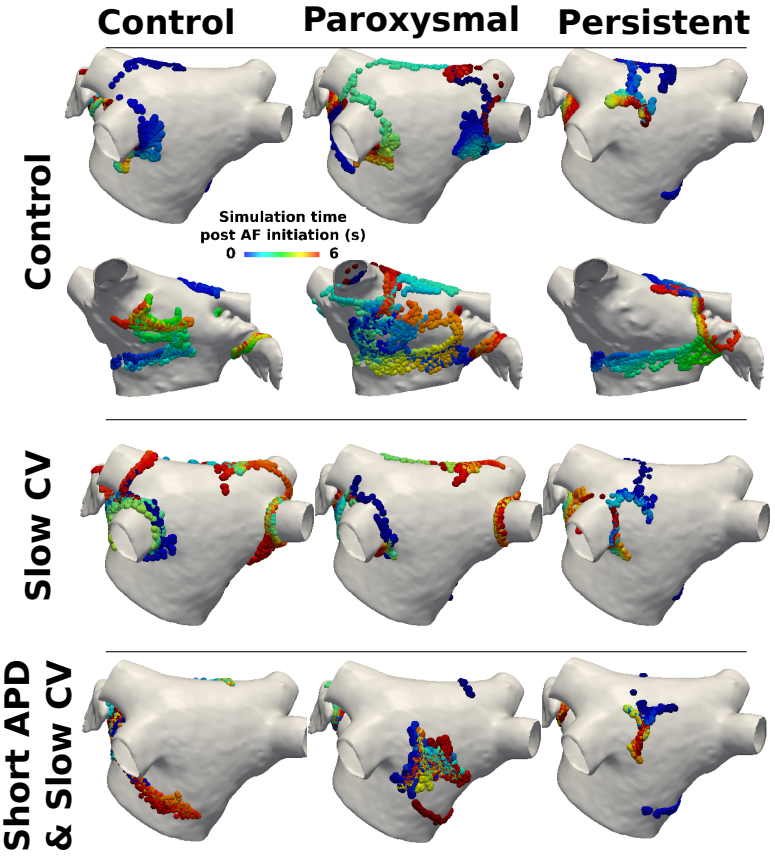

Figure 3. Phase singularity distributions showing that rotors are located at the LA/PV junction in the case of baseline PV APD but not for shorter PV APD, and stabilise to regions of high fibrosis density in persistent AF.

PV APD but not for shorter PV APD. Modifying PV CV did not affect whether LA/PV reentry is observed. Rotors were found to stabilise to regions of high fibrosis density in persistent $\mathrm{AF}$.

The relationship between LA fibrosis and PV properties on driver location was investigated on an individual patient basis for four patients. For patients for whom rotors were located away from the PVs (Fig 4 LA1), increasing model fibrosis from low to high increased the model agreement with clinical PS density $2.3 \pm 1.0$ fold. For other patients, lower levels of fibrosis were more appropriate (2.1 fold increase in agreement for lower fibrosis), and PV isolation converted fibrillation to macro reentry in the model (Fig 4 LA2).

\section{Discussion}

This simulation study investigated the effects of PV properties on arrhythmia inducibility, and the individual and combined effects of fibrosis and PV properties on driver location, based on population or individual patient data. Short PV ERP increased arrhythmia susceptibility; while longer PV ERP was found to be protective. Arrhythmia dynamics depend on both PV properties and fibrosis distribution, varying from meandering rotors to PV reenty to stable rotors at regions of high fibrosis density. Mea- 


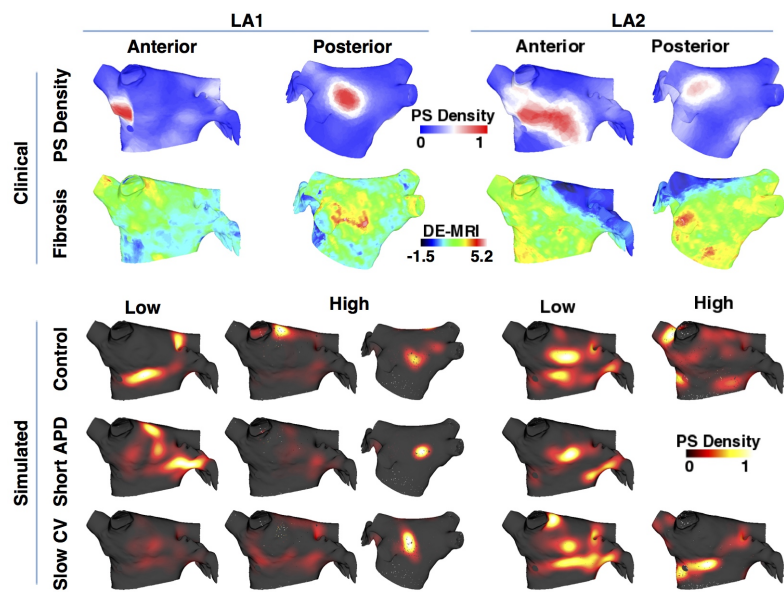

Figure 4. Clinical fibrosis and PS density maps for two patients, together with model PS density maps for cases with a low or high level of fibrosis, together with baseline PV properties, short PV APD or slow PV CV.

surement of fibrosis and PV properties may indicate patient specific susceptibility to AF initiation and maintenance.

\section{Acknowledgements}

This work was supported by the Lefoulon-Delalande Foundation, and by grants Equipex MUSIC ANR-11EQPX-0030, IHU LIRYC ANR-10-IAHU-04. Computer time was provided by MCIA (Mesocentre de Calcul Intensif Aquitain) of the Universite de Bordeaux and of the Universite de Pau et des Pays de l'Adour.

\section{References}

[1] Pascale P, Shah AJ, Roten L, Scherr D, Komatsu Y, Ramoul K, Daly M, Denis A, Derval N, Sacher F, et al. Pulmonary veins to left atrium cycle length gradient predicts procedural and clinical outcomes of persistent atrial fibrillation ablation. Circulation Arrhythmia and Electrophysiology 2014; CIRCEP-113.

[2] Jaïs P, Hocini M, Macle L, Choi KJ, Deisenhofer I, Weerasooriya R, Shah DC, Garrigue S, Raybaud F, Scavee C, et al. Distinctive electrophysiological properties of pulmonary veins in patients with atrial fibrillation. Circulation 2002;106(19):2479-2485.

[3] Hocini M, Ho SY, Kawara T, Linnenbank AC, Potse M, Shah D, Jaïs P, Janse MJ, Haïssaguerre M, De Bakker JM. Electrical conduction in canine pulmonary veins. Circulation 2002;105(20):2442-2448.

[4] Lim HS, Hocini M, Dubois R, Denis A, Derval N, Zellerhoff S, Yamashita S, Berte B, Mahida S, Komatsu Y, et al. Complexity and distribution of drivers in relation to duration of persistent atrial fibrillation. Journal of the American College of Cardiology 2017;69(10):1257-1269.

[5] Haissaguerre M, Shah AJ, Cochet H, Hocini M, Dubois R, Efimov I, Vigmond E, Bernus O, Trayanova N. Intermittent drivers anchoring to structural heterogeneities as a major pathophysiological mechanism of human persistent atrial fibrillation. The Journal of physiology 2016;594(9):23872398.

[6] Sohns C, Lemes C, Metzner A, Fink T, Chmelevsky M, Maurer T, Budanova M, Solntsev V, Schulze WH, Staab $\mathrm{W}$, et al. First-in-man analysis of the relationship between electrical rotors from noninvasive panoramic mapping and atrial fibrosis from magnetic resonance imaging in patients with persistent atrial fibrillation. Circulation Arrhythmia and Electrophysiology 2017;10(8):e004419.

[7] Labarthe S, Bayer J, Coudière Y, Henry J, Cochet H, Jaïs P, Vigmond E. A bilayer model of human atria: mathematical background, construction, and assessment. Europace 2014; 16(suppl_4):iv21-iv29.

[8] Krueger MW, Dorn A, Keller DU, Holmqvist F, Carlson J, Platonov PG, Rhode KS, Razavi R, Seemann G, Dössel O. In-silico modeling of atrial repolarization in normal and atrial fibrillation remodeled state. Medical biological engineering computing 2013;51(10):1105-1119.

[9] Haissaguerre M, Jaïs P, Shah DC, Takahashi A, Hocini M, Quiniou G, Garrigue S, Le Mouroux A, Le Métayer P, Clémenty J. Spontaneous initiation of atrial fibrillation by ectopic beats originating in the pulmonary veins. New England Journal of Medicine 1998;339(10):659-666.

[10] Verheule S, Wilson EE, Arora R, Engle SK, Scott LR, Olgin JE. Tissue structure and connexin expression of canine pulmonary veins. Cardiovascular research 2002;55(4):727738.

[11] Ho S, Cabrera J, Tran V, Farre J, Anderson R, SanchezQuintana D. Architecture of the pulmonary veins: relevance to radiofrequency ablation. Heart 2001;86(3):265-270.

[12] Bayer JD, Roney CH, Pashaei A, Jaïs P, Vigmond EJ. Novel radiofrequency ablation strategies for terminating atrial fibrillation in the left atrium: a simulation study. Frontiers in physiology 2016;7.

[13] Roney CH, Bayer JD, Zahid S, Meo M, Boyle PM, Trayanova NA, Haïssaguerre M, Dubois R, Cochet H, Vigmond EJ. Modelling methodology of atrial fibrosis affects rotor dynamics and electrograms. EP Europace 2016; 18(suppl_4):iv146-iv155.

[14] Haissaguerre M, Hocini M, Denis A, Shah AJ, Komatsu Y, Yamashita S, Daly M, Amraoui S, Zellerhoff S, Picat $\mathrm{MQ}$, et al. Driver domains in persistent atrial fibrillation. Circulation 2014; CIRCULATIONAHA-113.

[15] Cochet H, Mouries A, Nivet H, Sacher F, Derval N, Denis A, Merle M, Relan J, Hocini M, Haissaguerre M, et al. Age, atrial fibrillation, and structural heart disease are the main determinants of left atrial fibrosis detected by delayedenhanced magnetic resonance imaging in a general cardiology population. Journal of cardiovascular electrophysiology 2015;26(5):484-492.

Address for correspondence:

Dr Caroline H Roney

IHU-Liryc, Hôpital Xavier Arnozan, Avenue du Haut Lévèque, 33600 Pessac, France

caroline.roney@gmail.com 